\title{
The Exploration and Practice of the Teaching Reform on the Direction of Perception and Control of Internet of Things
}

\author{
Jianqiang Wang \\ School of information engineering, Yulin University, Yulin, Shaanxi, China \\ 317950860@qq.com
}

\begin{abstract}
The modular curriculum system construction is a hot topic in recent research on the teaching reform of applied talents training, training the application ability of the starting point, change the knowledge input output ability, but the curriculum system construction and implementation process there are some constraints and obstacles. As a new specialty, there are many problems in the process of teaching implementation. To solve the above problems, based on the study of curriculum system of module, network engineering professional a professional direction, perception and control the direction of, put forward project driven modular teaching reform based on the specific networking project was divided into ability module, and the ability to target design, teaching content selection, teaching schedule, teaching evaluation and teaching material construction is discussed, the specific implementation plan and process are presented.
\end{abstract}

Keywords: Modular curriculum system; Internet of things engineering; Perception and control; Teaching reform; Project driven

\section{物联网工程专业 “感知与控制” 方向模块化教学 改革的探索与实践}

\author{
王建强 \\ （榆林学院 信息工程学院,陕西榆林 719000 )
}

摘要: 模块化课程体系构建是近年来应用型人才培养教学改革研究的热点, 其出发点为应用能力的培养, 变知识输入为 能力输出, 但该课程体系的构建及实施过程均存在一定制约因素和障碍。物联网工程专业作为一个新专业, 在教学实施过程 中也存在诸多问题。针对以上问题, 在对模块化课程体系研究的基础上, 对物联网工程专业的一个专业方向-------感知与控 制方向, 提出了基于项目驱动模块化教学改革, 对具体的物联网工程项目进行了能力模块的划分, 并从能力目标设计、教学 内容选取、教学进度安排、教学评价及教材建设等方面进行了论述，给出了具体的方案。

关键词: 模块化课程体系; 物联网工程专业; 感知与控制方向; 教学改革; 项目驱动

中图分类号: G420 文献标志码: A

引言

近年来，国内部分应用型本科院校引入了德国的应用科技大学的人才培养模式，该模式在国外已经被 公认为是一种有效的应用型人才培养模式。国内高校在进行模块化人才培养模式改革的实践中也取得了许 多成果, 国内最早引入该培养模式的高校, 已经整体构建了基于能力导向的模块化教学体系, 以教学内容 模块化为核心, 更加的注重人才的能力培养, 将传统课堂的知识输入为导向改为以能力输出为导向; 对教 学内容、教学方式方法及教学环境等进行了进一步的优化, 以适应这种教学体系; 这种全校规模的教学体 
系构建完成后, 使得全校上下整体的教育理念有了根本的转变, 课程改革及教材建设等方面都有了进一步 的突破, 受教育者的能力有了进一步的发展。 $[1,2,3]$ 除此之外, 也有一些一线的高等教育工作者, 对某 一门具体的课程提出了模块化教学改革的方案并进行了探索, 在应用型人才的能力培养方面取得了一下宝 贵的经验。 $[4,5,6,7]$ 同时, 部分学者在采用项目驱动法进行教学改革的实践中, 也取得了一些成果。 $[8$, 9, 10]

然而, 模块化课程体系的构建, 对大多数应用型的本科院校来说, 还是一个全新的事物, 要将整个专 业甚至整个学校进行模块化教学体系的实施, 还存在着教育理念难转变、制度保障跟不上、教育资源受约 束等诸多难题 [3]。本文在分析了物联工程专业的一个专业方向当前教学过程中存在问题的基础上, 提出 先在该专业方向上小规模的实施模块化教学改革, 利用物联网工程的具体项目做支撑, 对具体工程项目进 行能力模块的分解, 并从能力目标设计、教学内容选取、教学进度安排、教学评价及教材建设等方面进行 了论述，给出了具体的教学改革实施方案。

\section{1 模块化教学体系构建及实施中面临的问题}

模块化教学体系有诸多的优点, 如培养目标更加明确、培养过程更加透明、教学安排灵活、教学方法 更加开放、学习效果更加突出、更有利于实践教学的实施等, 但模块化教学在构建及实施的过程中也存在 诸多难题。

\section{1 教育理念难转变}

国内高等教育走过了几十年的时间，人才培养模式也一直在改变，自 2014 年国家提出了应用型大学 转型以来, 人才培养模式的改革一直在进行, 但传统的教学模式在高校、教育者及被教育者的思想中, 根 深蒂固, 很难一下子被扭转。人才培养模式是高校培养人才的核心体现, 而教学体系又是人才培养模式的 核心内容, 学校在实施人才培养模式改革的过程中, 势必改变已有的教学内容、教学方式、教学手段等等, 很难保证所有的参与者能快速的把理念转变过来。

\section{2 制度保障跟不上}

一种全新的人才培养模式的实施，一个全新的教学体系的构建 ，离不开新的制度保障，大多数应用 型本科院校, 现行的政策和管理制度是在学科型教学体系的条件下制订的, 在实施模块化教学体系的过程 中, 需要从政策和制度上予以保证。为了确保模块化教学的总体质量, 需要制定相应的教学质量保障制度, 并形成完整的制度体系。包括模块教学质量保障制度、学生学习管理保障制度、实践教学保障制度和学生 创新能力保障制度等都需要进行全新的修改, 同样的, 对模块规划、实施及评价的制度也应被相应的制定, 如果不能出台合理的制度，将很难保证该教学体系的教学质量。[3]

\section{3 教育资源受约束}

模块化教学体系的制定和实施, 离不开教学资源。教师是实施模块化教学改革的主体, 在实施过程中 需要大量的理论基础扎实、较高的教学能力, 尤其是丰富的实践经验, 而大多数的高校, 双师型的人才少 之又少; 新的教学方式方法, 也将对教学场地, 实践环境等提出新的要求, 短时间内, 这些条件很难齐备。

\section{2 物联网工程专业感知与控制方向课程教学存在问题}

物联网工程是一个新上专业, 学科交叉是该专业的一个显著特点, 在几年的教学实践中, 我们发现在 课程设置及教材选用等方面都存在一些问题。 


\section{1 课程设置与编排不够合理}

作为一个新兴的交叉学科, 在最初的课程设置时, 不可避免的采用拼凑的方式来设置课程, 个别课程 的教学大纲也是直接套用其他学科专业的, 这样做的结果是课程的内容不能体现人才培养定位里边的能力 目标。部分课程理论性太强, 基本没有实践内容, 如传感器原理、RFID 原理、无线传感网络等课程, 对于 本专业来说，应该以应用为主，原理可以尽可能的少涉及。

\section{2 课程之间内容脱节, 部分课程之间内容重复}

无线传感网络课程里边的 Zigbee 协议栈实现的 SOC 应为无线 SOC，但目前我们的单片机课程仍以 STC89C52 来讲解, 而到了无线传感网络课程里边, 又没有关于 SOC 的教学内容, 使得教学内容脱节。个别 课程, 由于在设置的时候没有通盘考虑, 又存在着一些重复。这些问题都使得课程设置的质量不高, 影响 教学效果。

\section{3 基于项目驱动法的模块化教学改革及教学案例应用}

\section{1 项目构建}

我们在物联网工程专业感知与控制方向的模块化教学中, 选取了几个实际的物联网工程的实例, 如智 能家居系统、智慧农业系统、远程医疗等。这些例子都不同程度的将物联网的三个层次（感知层、传输层、 应用层）进行了应用。因此，我们选取这样的项目应用在教学中。

\section{2 能力模块划分及能力目标设计}

能力模块划分见表 1 。

表 1 能力模块划分表

\begin{tabular}{|c|c|c|}
\hline 能力模块名称 & 模块功能 & 知识点 \\
\hline $\begin{array}{l}\text { 感知层模块 } 1 \\
\text { 感知层模块 } 2\end{array}$ & $\begin{array}{c}\text { RFID 射频识别 } \\
\text { 通过传感器, 获取物理量 }\end{array}$ & $\begin{array}{l}\text { RFID 原理、应用, 与 CC2530 的连接电路 } \\
\text { 传感器原理、应用, 与 } \mathrm{CC} 2530 \text { 的连接电路 }\end{array}$ \\
\hline 感知层模块 3 & $\begin{array}{c}\text { CC2530 获取传感器及 RFID } \\
\text { 的信息 }\end{array}$ & $\begin{array}{l}\text { CC2530GPI0 口驱动、CC2530 串口驱动、中 } \\
\text { 断、时钟使用、CC2530 的原理及编程 }\end{array}$ \\
\hline 传输层模块 1 & $\begin{array}{c}\text { 通过 Zigbee 实现终端与协 } \\
\text { 调器的通信 }\end{array}$ & $\begin{array}{l}\text { Zigbee 协议概念、结构体系, Zigbee 组网, } \\
\text { Z-Stack 协议栈的体系结构, Z-Stack 协议 } \\
\text { 栈应用, 协议栈读取传感器物理量。 }\end{array}$ \\
\hline 块 2 & $\begin{array}{c}\text { 协调器模块与 S3c2440 (装 } \\
\text { 有嵌入式 Linux 操作系统) } \\
\text { 通过串口通信 }\end{array}$ & $\begin{array}{c}\text { CC2530 串口通信, S3c2440 硬件相关原理, } \\
\text { 嵌入式 Linux 开发环境搭建, 嵌入式 Linux } \\
\text { 移植, 嵌入式 Linux 驱动 S3c2440 的串口与 } \\
\text { 其他设备通信, }\end{array}$ \\
\hline 块 3 & $\begin{array}{l}\text { S3c2440(装有嵌入式Linux } \\
\text { 操作系统) 通过wifi 模块 } \\
\text { 与安卓设备通信 }\end{array}$ & $\begin{array}{c}\text { 嵌入式 Linux 驱动程序 (wifi 模块) 在 } \\
\text { S3c2440 上的移植, 无线路由器操作系统的 } \\
\text { 实现, 嵌入式 Linux 下网络套接字编程, 嵌 } \\
\text { 入式 Linux 下 Web 服务器的移植 }\end{array}$ \\
\hline 应 & $\begin{array}{l}\text { 安卓设备编程实现对无线 } \\
\text { 传感网络的检测及控制 }\end{array}$ & $\begin{array}{l}\text { 安卓开发应用, 安卓开发环境的搭建, 安卓 } \\
\text { 下的网络套接字编程, 安卓程序界面的设 } \\
\text { 计, 安卓下控制程序、界面显示程序的设计 }\end{array}$ \\
\hline
\end{tabular}

\section{3 教学内容选取及教材建设}

在教学内容的选取上, 我们一方面要遵循 “用到什么讲什么” 的原则, 根据项目能力模块的划分, 给 学生进行相应的知识点的讲解, 其余的内容暂时少讲甚至不讲, 待整改项目实施完成后, 根据时间安排, 对其余的内容进行选讲。

在教材选取方面, 由于物联网工程专业的特点, 且基于项目驱动的教学改革对本专业方向来说是一次 
全新的尝试, 故适合的教材基本没有, 我们采用自编教材或者选用国内现阶段比较好的一些校外培训机构 的教材，这样以来，教材的内容就可以适合于我们的教学实践。

\section{4 结束语}

在模块化教学体系的构建中，模块分为宏观、中观、微观三个层面，宏观模块描述一个完整专业学生 须获得的知识、能力和素质; 中观模块则为某一专业方向的学习重点及教学方案; 微观模块是整个模块的 基石, 描述的是每一个模块的具体内容, 并以其所要培养的知识、能力和素质满足中观和宏观模块所要达 到的能力要求. [11] 由于要构建一个宏观的模块, 现阶段来看, 实施起来困难较多, 因此在本次实践中, 我们构建的是物联网工程专业的一个专业方向-----感知与控制方向的模块化教学方案, 整个方案的实施 难度较低, 可作为同类院校在模块化教学改革的参考。

\section{5 致谢}

本文为榆林学院教学改革研究资助项目的阶段性成果。

\section{Acknowledgement}

This paper is the result of the research project of Yulin University teaching reform. 参考文献:

[1] 郡一江, 刘红. 基于能力导向的模块化教学体系构建一一合肥学院模块化教学改革的理论与实践 [J]. 合肥学院学 报 (自然科学版), 2013, (04): 58-63.

[2] 袁笈, 许强, 王晓峰, 等. 基于应用型人才培养的模块化教学改革研究一借鉴德国 FH 成功经验 $[J]$. 合肥学院 学报( 自然科学版)，2011，21（4)：56-65.

[3] 曹中红, 魏朱宝. 能力本位的应用型人才培养体系重构一一合肥学院会计专业模块化改革探索 [J]. 合肥学院学报 (自然科学版), 2011, (04):61-65.

[4] 许泽银, 徐强, 蒙争争. 机械设计制造及自动化专业模块化教学改革与实践 [J]. 合肥学院学报 (自然科学 版), 2015, (02) : 78-81.

[5] 刘海军, 商铁林. PLC 课程模块化教学改革实践 [J]. 榆林学院学报, 2015, (02) : 70-72.

[6] 李家明. 地方本科院校无机化学课程模块化教学改革探索 [J]. 钦州学院学报, 2015, (11) :24-28.

[7] 陈萌,鲍淑娣, 童春芽. 基于 MOOC 的公共计算机模块化教学改革研究 [J]. 宁波工程学院学报, 2014, (04) : 86-91.

[8] 刘献君，吴洪富. 人才培养模式改革的内涵、制约与出路 [J]. 中国高等教育， 2009（12）：10-13.

[9] 林元乘. 项目驱动法在计算机网络实验教学中的应用 [J]. 实验科学与技术，2011，9（4)：112-116.

[10］彭远芳, 陆勤,胡宁, 等. 基于 “项目驱动法” 的单片机课程一体化教学的探索与实践 [J]. 计算机教育, 2010（6）: $78-82$.

[11] 蔡敬民, 陈啸. 基于能力导向的模块化教学体系构建一一以合肥学院为例 [M]. 合肥: 中国科学技术大学出版社, 2012: 9 .

\section{References}

[1] Shao Yijiang, Liu Hong. Construction of modular teaching system based on ability oriented theory and practice of modular teaching reform in Hefei University [J]. Journal of Hefei University (NATURAL SCIENCE EDITION), 2013,(04):58-63. (in Chinese)

[2] Min Yuan, Xu Qiang, Wang Xiaofeng, et al. Study on Modular Teaching Reform of applied talents from Germany FH J. Based on the successful experience of Journal of Hefei University (NATURAL SCIENCE EDITION), 2011, 21 (4): 56 - 65 (in Chinese)

[3] Cao Zhonghong, Wei Zhu Bao. Reconstruction of competency based training system for Applied Talents: an exploration into the reform of Accounting Specialty in Hefei University [J]. Journal of Hefei University (NATURAL SCIENCE EDITION), 2011,(04):61-65. (in Chinese)

[4] Xu Zeyin, Xu Qiang, Zheng Zheng Meng. Mechanical design manufacturing and automation professional modular teaching reform and practice of $[\mathrm{J}]$. Journal of Hefei University (NATURAL SCIENCE EDITION), 2015, (02): 78-81. (in Chinese) 
[5] Liu Haijun, taking iron. PLC course reform of modular teaching practice [J]. Journal of Yulin University, 2015, (02): 70-72. (in Chinese)

[6] Li Ming. Modular inorganic chemistry teaching reform in local universities to explore [J]. Journal of Qinzhou University, 2015, (11): 24-28. (in Chinese)

[7] Chen Tong. Bao Shudi, Public computer module teaching reform based on MOOC [J]. Journal of Ningbo University of Technology, 2014, (04): 86-91. (in Chinese)

[8] Liu Xianjun, Wu Hongfu. Connotation, restriction and outlet of talent training mode reform [J]. Chinese higher education, 2009 (12): 10-13. (in Chinese)

[9] Lin Yuan Xiang. Application of project driven method in computer network experiment teaching [J]. Experimental science and technology, 2011, 9( 4) : 112-116. (in Chinese)

[10] Peng Yuanfang, Lu Qin, Hu Ning, et al. Exploration and practice of integrated teaching of single chip microcomputer based on project driven method [J]. Computer education, 2010 (6): 82. (in Chinese)

[11]Cai Jingmin, Chen Xiao. The modular construction of ability oriented teaching system -Taking Hefei University as an example [M] . Hefei University of Science \& Technology China press, 2012: 9 (in Chinese) 